OPEN ACCESS

Edited by:

Gerardo Ortiz,

Indiana University Bloomington,

United States

Reviewed by:

Orion Ciftja,

Prairie View A\&M University,

United States

Byungchan Han,

Yonsei University, South Korea

${ }^{*}$ Correspondence:

Giulio A. H. Schober

schober@physik.rwth-aachen.de

Specialty section:

This article was submitted to Condensed Matter Physics,

a section of the journal

Frontiers in Physics

Received: 24 October 2017

Accepted: 23 March 2018

Published: 08 May 2018

Citation:

Schober GAH, Ehrlich J, Reckling T and Honerkamp C (2018)

Truncated-Unity Functional

Renormalization Group for Multiband

Systems With Spin-Orbit Coupling.

Front. Phys. 6:32.

doi: 10.3389/fphy.2018.00032

\section{Truncated-Unity Functional Renormalization Group for Multiband Systems With Spin-Orbit Coupling}

\author{
Giulio A. H. Schober ${ }^{1 *}$, Jannis Ehrlich ${ }^{1,2}$, Timo Reckling ${ }^{1}$ and Carsten Honerkamp ${ }^{1,3}$ \\ ${ }^{1}$ Institute for Theoretical Solid State Physics, RWTH Aachen University, Aachen, Germany, ${ }^{2}$ Peter Grünberg Institute and \\ Institute for Advanced Simulation, Forschungszentrum Jülich, Jülich, Germany, ${ }^{3}$ JARA-FIT, Jülich Aachen Research Alliance - \\ Fundamentals of Future Information Technology, Jülich, Germany
}

Although the functional renormalization group (fRG) is by now a well-established method for investigating correlated electron systems, it is still undergoing significant technical and conceptual improvements. In particular, the motivation to optimally exploit the parallelism of modern computing platforms has recently led to the development of the "truncated-unity" functional renormalization group (TU-fRG). Here, we review this fRG variant, and we provide its extension to multiband systems with spin-orbit coupling. Furthermore, we discuss some aspects of the implementation and outline opportunities and challenges ahead for predicting the ground-state ordering and emergent energy scales for a wide class of quantum materials.

Keywords: functional renormalization group, interacting fermions, high-performance computing, multiband systems, spin-orbit coupling, quantum materials

\section{INTRODUCTION}

The rapid progress of condensed matter physics in recent decades, in its full breadth from fundamental science to technological applications, has mainly been based on the systematic development and strengthening of two main pillars: materials and methods.

On the one hand, the discovery of new classes of quantum materials such as high-temperature superconductors and topological insulators has brought forth a rich variety of new effects and astonishing phenomena (for recent reviews see e.g., [1-3]). Among others, spin-orbit coupling has been identified as an important player in the formation of exotic phases with unconventional bulk and surface properties [4,5]. Consequently, multiband systems with spin-orbit coupling (for some references, see e.g., [6]) are by now a major topic of cutting-edge research in condensed matter physics, which largely overlaps with the fields of spintronics and topological matter. Their study has led to new theoretical insights regarding, for example, relativistic effects in solids $[7,8]$ and emerging entities such as Majorana quasiparticles $[9,10]$. In addition, quantum materials with spin-orbit coupling hold the promise for technological applications ranging from highperformance, high-density memories to fault-tolerant topological information processing [11, 12]. More concretely, the Rashba model has become a paradigm for coupling the spin and orbital degrees of freedom [13]. While having traditionally been observed at surfaces or interfaces between different materials, more recently, $\mathrm{BiTeX}(\mathrm{X}=\mathrm{Cl}, \mathrm{Br}$, and $\mathrm{I})$ as well as GeTe have attracted much interest as giant bulk Rashba semiconductors [14-18]. Hence, these materials are also promising candidates for spintronics applications such as the Datta-Das spin transistor [19, 20]. In this context, we also mention the recent proposal [21] of a spintronic device which does not require the injection of spin-polarized electrons from one quantum dot to the other. 
On the other hand, exotic material behavior often originates from electronic correlations, and the quest for new computational methods capable of dealing with these correlations is a driving force in modern materials physics. While the most prominent example for a non-trivial interactioninduced quantum phase may be an ordinary superconductor, more recently, interaction-driven topological phases have also attracted much interest (see e.g., [22, 23]). In particular, the interplay between spin-orbit coupling and electron-electron interactions gives rise to a plethora of unconventional quantum effects such as mixed singlet-triplet superconductivity induced by the Rashba coupling $[6,24,25]$. However, simultaneously treating the enormous number of electrons in a solid requires the development of new computational approaches, which on the one hand capture the essential physics and on the other hand allow for practical calculations on reasonable time scales. Fortunately, the recent advancement of condensed matter science has come along with an equally steep increase in the available computational resources, which is mainly due to the evolution of building blocks of large computing architectures from single-core CPUs to compute nodes with multiple cores. This development naturally requires the adaption of modern calculation methods to optimally exploit the parallelism of modern computing platforms.

In this article, we summarize recent progress in the field of fRG for fermions in solids, which was achieved by the development of the truncated-unity functional renormalization group (TU-fRG) [26-28]. Moreover, we take one step forward by adapting this method for application to multiband systems with spin-orbit coupling.

To begin with, the functional renormalization group (fRG) is a field-theoretical approach to the electronic many-body problem, which is capable of treating the different energy scales in the electronic spectrum of a solid as well as the different-and possibly competing-ordering tendencies at low temperatures [29-31]. Having undergone various phases of exploration and refinement, it is by now regarded as an unbiased method with the potential of reaching quantitative precision in the prediction of energy scales and parameter ranges [26]. The different energy scales are treated by successively "integrating out" the highenergy degrees of freedom in the path integral formalism and thereby deriving effective interactions for the low-energy degrees of freedom. In a solid, the latter correspond to the electrons at the Fermi level, hence the fRG can predict the ground state ordering of the many-electron system. As compared to exact methods such as lattice quantum Monte Carlo (QMC) simulations, the fRG has the advantage of not being limited strongly in the choice of tight-binding and interaction parameters (which is necessary in the case of QMC to avoid the occurrence of a sign problem) [32]. Until recently, the fRG was usually combined with a Fermi surface patching approximation, whereby it has been successfully applied to various material classes such as high-temperature superconductors [33-35], mono- and few-layer graphene [3639], and systems with spin-orbit coupling [6, 40, 41]. For recent reviews of the fRG in solid-state physics, we refer the interested reader to Schober et al. [6], Metzner et al. [30], and Platt et al. [31].
As a recent variant of the $\mathrm{fRG}$ technique, the TU-fRG has been developed and applied to extended Hubbard models on the square and honeycomb lattices [26-28, 32]. This method is based on earlier approaches called the exchange parametrization $f R G$ [42] (see also [43-45]) and the singular-mode fRG (SM-fRG) [46]. As compared to the exchange parametrization fRG for the twofermion interaction, the TU-fRG introduces additional insertions of truncated partitions of unity, which decouple the fermionic propagators from the exchange propagators. This leads to a separation of the underlying differential equations and therefore enables an efficient parallelization on a large number of compute nodes (for details on the numerical implementation, see [26, 28, 32]). Remarkably, in the TU-fRG implementation, the CPU time scales only linearly with the momentum resolution of the twofermion vertex as compared to a quartic dependence in the Fermi surface patching approximation. Therefore, the TU-fRG allows one to capture the wavevector dependence of the effective twofermion vertices with an unrivaled precision [26], thus paving the way for quantitative predictions of phase diagrams, leading correlations and emergent energy scales in multiband systems with realistic $a b$ initio interaction parameters. This procedure has already been demonstrated in Sánchez de la Peña et al. [28] for the case of graphene. Most recently, it has even been applied in parameter ranges which are not accessible to QMC simulations [32].

So far, the application of the TU-fRG has been restricted to spin-SU(2)-invariant systems. In fact, this symmetry is exploited in the very derivation of the flow equations [26] (see also [47]). Since this restriction excludes the important class of materials with spin-orbit coupling, it is highly desirable to derive a generalization of the TU-fRG which does not assume the $\mathrm{SU}(2)$ symmetry of the single-particle Hamiltonian. In this generalization, the two-fermion vertices will not only acquire additional spin indices, but also the general structure of the flow equations will be modified. Apart from providing a general perspective on the TU-fRG technique, it is precisely the goal of the present article to derive such generalized flow equations. In order to keep the formalism simpler we will not discuss the treatment of the frequency dependence of the interactions (for a recent treatment, see $[48,49])$. Furthermore, we will not consider self-energy corrections on the internal lines (for some discussion of this aspect, see $[30,48,49])$. We remark that in the SMfRG context, generalized flow equations for non-SU(2)-invariant systems have already been derived [50] and successfully applied to various materials with spin-orbit coupling [51-53].

This article is organized as follows: In section 2, we define the projections of an arbitrary two-particle vertex onto the particleparticle, crossed particle-hole, and direct particle-hole channels. In section 3, we first define the exchange propagators from the respective single-channel coupling functions. Subsequently, we derive the flow equations for these exchange propagators, and we show that the number of relevant equations can be reduced by using an antisymmetry relation between crossed and direct particle-hole terms. Finally, the set of TU-fRG equations is completed by the cross projections between the particle-particle and particle-hole terms, for which we also provide explicit expressions. In section 4 , we derive the transformation laws for 
the exchange propagators between the orbital (or spin) and the band basis, and we briefly compare these two representations with respect to the numerical effort required to solve the flow equations. In section 5, we benchmark the generalized TU-fRG equations by applying them to the Rashba model with a local interaction. Restricting us to the particle-particle channel, we provide an analytic solution which is consistent with the ladder resummation of Schober et al. [6]. Finally, we give an outlook on future applications of the TU-fRG to realistic multiband models with spin-orbit coupling.

\section{CHANNEL DECOMPOSITION}

We put our main focus on the fRG for the two-particle vertex $V$ (see e.g., [47]). The renormalization group equation (RGE) for this vertex is an ordinary differential equation with respect to a scaling parameter $\Lambda$, which has contributions from three different channels: particle-particle, crossed particle-hole, and direct particle-hole channel (see section 3). An analysis as well as previous numerical investigations reveal that each channel has a strong dependence on one particular momentum contribution [30]. Therefore, we define the functionals $\hat{P}[V], \hat{C}[V]$, and $\hat{D}[V]$ as projections onto these channels, which are parametrized by their respective main momentum such that the following equations hold (we use similar conventions as in Eckhardt et al. [54]):

$$
\begin{aligned}
V\left(\boldsymbol{k}_{1}, \boldsymbol{k}_{2}, \boldsymbol{k}_{3}\right) & =\sum_{\boldsymbol{R}, \boldsymbol{R}^{\prime}} \hat{P}[V]_{\boldsymbol{R} \boldsymbol{R}^{\prime}}\left(\boldsymbol{k}_{1}+\boldsymbol{k}_{2}\right) f_{\boldsymbol{R}}^{*}\left(\boldsymbol{k}_{1}\right) f_{\boldsymbol{R}^{\prime}}\left(\boldsymbol{k}_{4}\right), \\
V\left(\boldsymbol{k}_{1}, \boldsymbol{k}_{2}, \boldsymbol{k}_{3}\right) & =\sum_{\boldsymbol{R}, \boldsymbol{R}^{\prime}} \hat{C}[V]_{\boldsymbol{R} \boldsymbol{R}^{\prime}}\left(\boldsymbol{k}_{1}-\boldsymbol{k}_{3}\right) f_{\boldsymbol{R}}^{*}\left(\boldsymbol{k}_{1}\right) f_{\boldsymbol{R}^{\prime}}\left(\boldsymbol{k}_{4}\right), \\
V\left(\boldsymbol{k}_{1}, \boldsymbol{k}_{2}, \boldsymbol{k}_{3}\right) & =\sum_{\boldsymbol{R}, \boldsymbol{R}^{\prime}} \hat{D}[V]_{\boldsymbol{R} \boldsymbol{R}^{\prime}}\left(\boldsymbol{k}_{3}-\boldsymbol{k}_{2}\right) f_{\boldsymbol{R}}^{*}\left(\boldsymbol{k}_{1}\right) f_{\boldsymbol{R}^{\prime}}\left(\boldsymbol{k}_{3}\right) .
\end{aligned}
$$

Here, $\boldsymbol{k}_{1}$ and $\boldsymbol{k}_{2}$ are ingoing momenta, while $\boldsymbol{k}_{3}$ and $\boldsymbol{k}_{4} \equiv \boldsymbol{k}_{1}+$ $\boldsymbol{k}_{2}-\boldsymbol{k}_{3}$ are outgoing momenta of the vertex function (where momentum conservation follows from translational invariance). Furthermore, the form factors $f_{\boldsymbol{R}}(\boldsymbol{k})$ are labeled by an index $\boldsymbol{R}$, which may correspond to the (Bravais) lattice coordinates in the case of plane-wave functions, $f_{\boldsymbol{R}}(\boldsymbol{k})=\exp (-\mathrm{i} \boldsymbol{k} \cdot \boldsymbol{R})$. On the real lattice, the form factors then become bond selectors, $f_{\boldsymbol{R}}\left(\boldsymbol{R}^{\prime}\right)=$ $\delta_{R, R^{\prime}}$. This provides a very natural description of the interaction as the sum of interacting fermion bilinears of particle-particle $(P)$ or particle-hole type $(C, D)$ that live on bonds of length $\boldsymbol{R}$. In such a picture, the truncation of the sums in Equations (1-3) beyond a certain $\left|\boldsymbol{R}_{\mathrm{c}}\right|$ just means that interactions of bilinears with a range longer than $\left|\boldsymbol{R}_{\mathrm{c}}\right|$ are ignored. Note that this truncation in the pair length does not imply a restriction on the length of the pairwise interaction between the bilinears kept. Depending on the symmetry of the problem under consideration, it may be useful to form linear combinations of the form factors that transform according to a specific irreducible representation of the point group, as done in Lichtenstein et al. [26] or explained in Platt et al. [31]. More generally, we only assume that the form factors constitute an orthonormal basis in the following sense:

$$
\begin{aligned}
\frac{1}{N} \sum_{\boldsymbol{k}} f_{\boldsymbol{R}}(\boldsymbol{k}) f_{\boldsymbol{R}^{\prime}}^{*}(\boldsymbol{k}) & =\delta_{\boldsymbol{R}, \boldsymbol{R}^{\prime}}, \\
\sum_{\boldsymbol{R}} f_{\boldsymbol{R}}(\boldsymbol{k}) f_{\boldsymbol{R}}^{*}\left(\boldsymbol{k}^{\prime}\right) & =N \delta_{\boldsymbol{k}, \boldsymbol{k}^{\prime}},
\end{aligned}
$$

where $N$ denotes the total particle number. We relabel the main momentum transfers as $\boldsymbol{s}=\boldsymbol{k}_{1}+\boldsymbol{k}_{2}, \boldsymbol{u}=\boldsymbol{k}_{1}-\boldsymbol{k}_{3}$, and $\boldsymbol{t}=\boldsymbol{k}_{3}-\boldsymbol{k}_{2}$, such that Equations (1-3) are equivalent to

$$
\begin{aligned}
V\left(\boldsymbol{k}, \boldsymbol{s}-\boldsymbol{k}, \boldsymbol{s}-\boldsymbol{k}^{\prime}, \boldsymbol{k}^{\prime}\right) & =\sum_{\boldsymbol{R}, \boldsymbol{R}^{\prime}} f_{\boldsymbol{R}}^{*}(\boldsymbol{k}) f_{\boldsymbol{R}^{\prime}}\left(\boldsymbol{k}^{\prime}\right) \hat{P}[V]_{\boldsymbol{R} \boldsymbol{R}^{\prime}}(\boldsymbol{s}), \\
V\left(\boldsymbol{k}, \boldsymbol{k}^{\prime}-\boldsymbol{u}, \boldsymbol{k}-\boldsymbol{u}, \boldsymbol{k}^{\prime}\right) & =\sum_{\boldsymbol{R}, \boldsymbol{R}^{\prime}} f_{\boldsymbol{R}}^{*}(\boldsymbol{k}) f_{\boldsymbol{R}^{\prime}}\left(\boldsymbol{k}^{\prime}\right) \hat{C}[V]_{\boldsymbol{R} \boldsymbol{R}^{\prime}}(\boldsymbol{u}), \\
V\left(\boldsymbol{k}, \boldsymbol{k}^{\prime}-\boldsymbol{t}, \boldsymbol{k}^{\prime}, \boldsymbol{k}-\boldsymbol{t}\right) & =\sum_{\boldsymbol{R}, \boldsymbol{R}^{\prime}} f_{\boldsymbol{R}}^{*}(\boldsymbol{k}) f_{\boldsymbol{R}^{\prime}}\left(\boldsymbol{k}^{\prime}\right) \hat{D}[V]_{\boldsymbol{R} \boldsymbol{R}^{\prime}}(\boldsymbol{t}),
\end{aligned}
$$

where, for the sake of clarity, we have written out explicitly the fourth momentum argument of the vertex function (which is determined by momentum conservation). The converse equations, which can be regarded as explicit definitions of the functionals (projections onto the different channels) $\hat{P}[V], \hat{C}[V]$, and $\hat{D}[V]$, then read as follows:

$$
\begin{aligned}
& \hat{P}[V]_{R R^{\prime}}(\boldsymbol{s})=\frac{1}{N^{2}} \sum_{\boldsymbol{k}, \boldsymbol{k}^{\prime}} f_{\boldsymbol{R}}(\boldsymbol{k}) f_{\boldsymbol{R}^{\prime}}^{*}\left(\boldsymbol{k}^{\prime}\right) V\left(\boldsymbol{k}, \boldsymbol{s}-\boldsymbol{k}, \boldsymbol{s}-\boldsymbol{k}^{\prime}, \boldsymbol{k}^{\prime}\right), \\
& \hat{C}[V]_{\boldsymbol{R} \boldsymbol{R}^{\prime}}(\boldsymbol{u})=\frac{1}{N^{2}} \sum_{\boldsymbol{k}, \boldsymbol{k}^{\prime}} f_{\boldsymbol{R}}(\boldsymbol{k}) f_{\boldsymbol{R}^{\prime}}^{*}\left(\boldsymbol{k}^{\prime}\right) V\left(\boldsymbol{k}, \boldsymbol{k}^{\prime}-\boldsymbol{u}, \boldsymbol{k}-\boldsymbol{u}, \boldsymbol{k}^{\prime}\right), \\
& \hat{D}[V]_{\boldsymbol{R} \boldsymbol{R}^{\prime}}(\boldsymbol{t})=\frac{1}{N^{2}} \sum_{\boldsymbol{k}, \boldsymbol{k}^{\prime}} f_{\boldsymbol{R}}(\boldsymbol{k}) f_{\boldsymbol{R}^{\prime}}^{*}\left(\boldsymbol{k}^{\prime}\right) V\left(\boldsymbol{k}, \boldsymbol{k}^{\prime}-\boldsymbol{t}, \boldsymbol{k}^{\prime}, \boldsymbol{k}-\boldsymbol{t}\right) .
\end{aligned}
$$

The conventions that we have chosen here have the following advantages (compare $[26,46])$ :

1. Each form factor in Equations (1-3) depends on only one momentum, which is part of the original vertex. This will facilitate the following derivations.

2. If two vertices fulfill $V\left(\boldsymbol{k}_{1}, \boldsymbol{k}_{2}, \boldsymbol{k}_{3}, \boldsymbol{k}_{4}\right)=-W\left(\boldsymbol{k}_{1}, \boldsymbol{k}_{2}, \boldsymbol{k}_{4}, \boldsymbol{k}_{3}\right)$, then

$$
\hat{D}[V]_{R R^{\prime}}(\boldsymbol{t})=-\hat{C}[W]_{R R^{\prime}}(\boldsymbol{t}) .
$$

In particular, if the vertex $V$ is antisymmetric with respect to its outgoing momenta such that $V\left(\boldsymbol{k}_{1}, \boldsymbol{k}_{2}, \boldsymbol{k}_{3}, \boldsymbol{k}_{4}\right)=$ $-V\left(\boldsymbol{k}_{1}, \boldsymbol{k}_{2}, \boldsymbol{k}_{4}, \boldsymbol{k}_{3}\right)$, then

$$
\hat{D}[V]=-\hat{C}[V]
$$

These identities will become crucial in section 3.2.

3. If $V$ is hermitian in the sense that $V\left(\boldsymbol{k}_{1}, \boldsymbol{k}_{2}, \boldsymbol{k}_{3}, \boldsymbol{k}_{4}\right)=$ $V^{*}\left(\boldsymbol{k}_{4}, \boldsymbol{k}_{3}, \boldsymbol{k}_{2}, \boldsymbol{k}_{1}\right)$, then

$$
\hat{P}[V]_{R R^{\prime}}(\boldsymbol{s})=\hat{P}[V]_{\boldsymbol{R}^{\prime} \boldsymbol{R}}^{*}(\boldsymbol{s}),
$$


i.e., the matrix $\hat{P}[V](s)$ is also hermitian for any $s$. The same applies to the matrix $\hat{C}[V](\boldsymbol{u})$, and to $\hat{D}[V](\boldsymbol{t})$ provided that $V\left(\boldsymbol{k}_{1}, \boldsymbol{k}_{2}, \boldsymbol{k}_{3}, \boldsymbol{k}_{4}\right)=V\left(\boldsymbol{k}_{2}, \boldsymbol{k}_{1}, \boldsymbol{k}_{4}, \boldsymbol{k}_{3}\right)$.

Finally, we remark that by definition all wavevectors including $\boldsymbol{s}, \boldsymbol{u}, \boldsymbol{t}$ are restricted to the first Brillouin zone. For example, Equation (1) is a shorthand notation for

$$
V\left(\boldsymbol{k}_{1}, \boldsymbol{k}_{2}, \boldsymbol{k}_{3}\right)=\sum_{\boldsymbol{R}, \boldsymbol{R}^{\prime}} f_{\boldsymbol{R}}^{*}\left(\boldsymbol{k}_{1}\right) f_{\boldsymbol{R}^{\prime}}\left(\boldsymbol{k}_{4}\right) \sum_{\boldsymbol{s}} \sum_{\boldsymbol{K}} \delta_{\boldsymbol{s}+\boldsymbol{K}, \boldsymbol{k}_{1}+\boldsymbol{k}_{2}} \hat{P}[V]_{\boldsymbol{R} \boldsymbol{R}^{\prime}}(\boldsymbol{s}) .
$$

Here, we formally sum over all reciprocal lattice vectors $\boldsymbol{K}$, but the condition that $\boldsymbol{k}_{1}, \boldsymbol{k}_{2}$, and $\boldsymbol{s}$ all lie in the first Brillouin zone fixes precisely one vector $\boldsymbol{K}$ which gives a non-vanishing contribution.

\section{RENORMALIZATION GROUP EQUATIONS}

\subsection{Derivation}

We now consider a general multiband system, where the vertex additionally depends on four band indices, which we denote by Latin letters. (By contrast, orbital or spin indices will be denoted by Greek letters, see section 4.) In the general case without $S U(2)$ symmetry, the RGE in the band basis reads as follows (see [47], or [6, Equations (82-89)]):

$$
\begin{aligned}
& \frac{\mathrm{d}}{\mathrm{d} \Lambda}\left(V_{\Lambda}\right)_{n_{1} n_{2} n_{3} n_{4}}\left(\boldsymbol{p}_{1}, \boldsymbol{p}_{2}, \boldsymbol{p}_{3}\right)= \\
& {\left[\Phi_{\Lambda}^{\mathrm{pp}}+\Phi_{\Lambda}^{\mathrm{ph}, \mathrm{c}}+\Phi_{\Lambda}^{\mathrm{ph}, \mathrm{d}}\right]_{n_{1} n_{2} n_{3} n_{4}}\left(\boldsymbol{p}_{1}, \boldsymbol{p}_{2}, \boldsymbol{p}_{3}\right)}
\end{aligned}
$$

where the particle-particle, crossed particle-hole, and direct particle-hole terms are given by (suppressing $\Lambda$ dependencies in the notation).

$$
\begin{aligned}
& \Phi_{n_{1} n_{2} n_{3} n_{4}}^{\mathrm{pp}}\left(\boldsymbol{p}_{1}, \boldsymbol{p}_{2}, \boldsymbol{p}_{3}\right)= \\
& -\frac{1}{N} \sum_{\ell_{1}, \ell_{2}} \sum_{\boldsymbol{k}} V_{n_{1} n_{2} \ell_{1} \ell_{2}}\left(\boldsymbol{p}_{1}, \boldsymbol{p}_{2}, \boldsymbol{k}\right) L_{\ell_{1} \ell_{2}}^{-}\left(\boldsymbol{k}, \boldsymbol{p}_{1}+\boldsymbol{p}_{2}-\boldsymbol{k}\right) \\
& V_{\ell_{1} \ell_{2} n_{3} n_{4}}\left(\boldsymbol{k}, \boldsymbol{p}_{1}+\boldsymbol{p}_{2}-\boldsymbol{k}, \boldsymbol{p}_{3}\right) \\
& \Phi_{n_{1} n_{2} n_{3} n_{4}}^{\mathrm{ph}, \mathrm{p}}\left(\boldsymbol{p}_{1}, \boldsymbol{p}_{2}, \boldsymbol{p}_{3}\right)= \\
& \frac{2}{N} \sum_{\ell_{1}, \ell_{2}} \sum_{\boldsymbol{k}} V_{n_{1} \ell_{2} n_{3} \ell_{1}}\left(\boldsymbol{p}_{1}, \boldsymbol{k}+\boldsymbol{p}_{3}-\boldsymbol{p}_{1}, \boldsymbol{p}_{3}\right) L_{\ell_{1} \ell_{2}}^{+}\left(\boldsymbol{k}, \boldsymbol{k}+\boldsymbol{p}_{3}-\boldsymbol{p}_{1}\right) \\
& V_{\ell_{1} n_{2} \ell_{2} n_{4}}\left(\boldsymbol{k}, \boldsymbol{p}_{2}, \boldsymbol{k}+\boldsymbol{p}_{3}-\boldsymbol{p}_{1}\right), \\
& \Phi_{n_{1} n_{2} n_{3} n_{4}}^{\mathrm{ph}, \mathrm{d}}\left(\boldsymbol{p}_{1}, \boldsymbol{p}_{2}, \boldsymbol{p}_{3}\right)=-\Phi_{n_{1} n_{2} n_{4} n_{3}}^{\mathrm{ph}, \mathrm{p}}\left(\boldsymbol{p}_{1}, \boldsymbol{p}_{2}, \boldsymbol{p}_{1}+\boldsymbol{p}_{2}-\boldsymbol{p}_{3}\right) .(19)
\end{aligned}
$$

Furthermore, the particle-particle loop $L^{-}$and the particle-hole loop $L^{+}$are defined as

$$
L_{\ell_{1} \ell_{2}}^{\mp}\left(\boldsymbol{k}_{1}, \boldsymbol{k}_{2}\right)=\frac{\mathrm{d}}{\mathrm{d} \Lambda}\left(\chi\left(e_{\ell_{1}}\left(\boldsymbol{k}_{1}\right)\right) \chi\left(e_{\ell_{2}}\left(\boldsymbol{k}_{2}\right)\right)\right) F_{\ell_{1} \ell_{2}}^{\mp}\left(\boldsymbol{k}_{1}, \boldsymbol{k}_{2}\right),
$$

with the functions $F_{\Lambda}^{\mp}$ given by

$$
\begin{aligned}
& F_{\ell_{1} \ell_{2}}^{-}\left(\boldsymbol{k}_{1}, \boldsymbol{k}_{2}\right)=\frac{1-f\left(e_{\ell_{1}}\left(\boldsymbol{k}_{1}\right)\right)-f\left(e_{\ell_{2}}\left(\boldsymbol{k}_{2}\right)\right)}{e_{\ell_{1}}\left(\boldsymbol{k}_{1}\right)+e_{\ell_{2}}\left(\boldsymbol{k}_{2}\right)}, \\
& F_{\ell_{1} \ell_{2}}^{+}\left(\boldsymbol{k}_{1}, \boldsymbol{k}_{2}\right)=\frac{f\left(e_{\ell_{1}}\left(\boldsymbol{k}_{1}\right)\right)-f\left(e_{\ell_{2}}\left(\boldsymbol{k}_{2}\right)\right)}{e_{\ell_{1}}\left(\boldsymbol{k}_{1}\right)-e_{\ell_{2}}\left(\boldsymbol{k}_{2}\right)} .
\end{aligned}
$$

Here, $e_{\ell}(\boldsymbol{k})=E_{\ell}(\boldsymbol{k})-\mu$ are the eigenvalues of the single-particle Hamiltonian measured relatively to the chemical potential, and $f(e)=\left(\mathrm{e}^{\beta e}+1\right)^{-1}$ denotes the Fermi distribution function. We have assumed a momentum regulator $\chi_{\Lambda}(e(\boldsymbol{k}))$, which suppresses all momenta inside a shell of thickness $\Lambda$ around the Fermi surfaces [30], but the above formulas can easily be generalized to other regulators (see e.g., [42]). Moreover, inherent in the above RGE is the level-two truncation, which neglects all Green functions with six or more external legs. Futher neglected are the self-energy and the frequency dependence of the four-point function. These approximations have already been applied successfully in many works before $[6,30,31]$.

We now define the scale-dependent single-channel coupling functions as follows (suppressing momentum dependencies to lighten the notation):

$$
\begin{aligned}
& V_{n_{1} n_{2} n_{3} n_{4}}^{(P)}(\Lambda):=\int_{\Lambda_{0}}^{\Lambda} \mathrm{d} \Lambda^{\prime} \Phi_{n_{1} n_{2} n_{3} n_{4}}^{\mathrm{pp}}\left(\Lambda^{\prime}\right), \\
& V_{n_{1} n_{2} n_{3} n_{4}}^{(C)}(\Lambda):=\int_{\Lambda_{0}}^{\Lambda} \mathrm{d} \Lambda^{\prime} \Phi_{n_{1} n_{2} n_{3} n_{4}}^{\mathrm{ph}, \mathrm{c}}\left(\Lambda^{\prime}\right), \\
& V_{n_{1} n_{2} n_{3} n_{4}}^{(D)}(\Lambda):=\int_{\Lambda_{0}}^{\Lambda} \mathrm{d} \Lambda^{\prime} \Phi_{n_{1} n_{2} n_{3} n_{4}}^{\mathrm{ph}, \mathrm{d}}\left(\Lambda^{\prime}\right),
\end{aligned}
$$

such that the scale-dependent vertex can be decomposed into four contributions,

$$
V(\Lambda)=V^{(0)}+V^{(P)}(\Lambda)+V^{(C)}(\Lambda)+V^{(D)}(\Lambda),
$$

where $V^{(0)} \equiv V\left(\Lambda_{0}\right)$ is the initial interaction. Furthermore, we define the exchange propagators as the following matrices (suppressing again $\Lambda$ dependencies):

$$
\begin{aligned}
& P_{n_{1} n_{2} n_{3} n_{4}}^{\boldsymbol{R \boldsymbol { R } ^ { \prime }}}(\boldsymbol{s}):=\hat{P}\left[V_{n_{1} n_{2} n_{3} n_{4}}^{(P)}\right]_{\boldsymbol{R \boldsymbol { R } ^ { \prime }}}(\boldsymbol{s}), \\
& C_{n_{1} n_{2} n_{3} n_{4}}^{\boldsymbol{R} \boldsymbol{R}^{\prime}}(\boldsymbol{u}):=\hat{C}\left[V_{n_{1} n_{2} n_{3} n_{4}}^{(C)}\right]_{\boldsymbol{R} \boldsymbol{R}^{\prime}}(\boldsymbol{u}), \\
& D_{n_{1} n_{2} n_{3} n_{4}}^{\boldsymbol{R} \boldsymbol{R}^{\prime}}(\boldsymbol{t}):=\hat{D}\left[V_{n_{1} n_{2} n_{3} n_{4}}^{(D)}\right]_{\boldsymbol{R} \boldsymbol{R}^{\prime}}(\boldsymbol{t}) .
\end{aligned}
$$

Per constructionem, a scale derivative acting on these matrices yields the following RGE:

$$
\begin{gathered}
\dot{P}_{n_{1} n_{2} n_{3} n_{4}}^{\boldsymbol{R \boldsymbol { R } ^ { \prime }}}(\boldsymbol{s})=\hat{P}\left[\Phi_{n_{1} n_{2} n_{3} n_{4}}^{\mathrm{pp}}\right]_{\boldsymbol{R} \boldsymbol{R}^{\prime}}(\boldsymbol{s}), \\
\dot{C}_{n_{1} n_{2} n_{3} n_{4}}^{\boldsymbol{R} \boldsymbol{R}^{\prime}}(\boldsymbol{u})=\hat{C}\left[\Phi_{n_{1} n_{2} n_{3} n_{4}}^{\mathrm{ph}, \mathrm{c}}\right]_{\boldsymbol{R} \boldsymbol{R}^{\prime}}(\boldsymbol{u}), \\
\dot{D}_{n_{1} n_{2} n_{3} n_{4}}^{\boldsymbol{R} \boldsymbol{R}^{\prime}}(\boldsymbol{t})=\hat{D}\left[\Phi_{n_{1} n_{2} n_{3} n_{4}}^{\mathrm{ph}, \mathrm{d}}\right]_{\boldsymbol{R} \boldsymbol{R}^{\prime}}(\boldsymbol{t}) .
\end{gathered}
$$

To obtain a closed system of differential equations, one further has to project the right-hand sides onto the form-factor basis. For 
this purpose, we insert partitions of unity of the form-factor basis [26],

$$
1=\sum_{\boldsymbol{q}} \delta_{\boldsymbol{q}, \boldsymbol{q}^{\prime}}=\frac{1}{N} \sum_{\boldsymbol{q}} \sum_{\boldsymbol{R}} f_{R}^{*}(\boldsymbol{q}) f_{\boldsymbol{R}}\left(\boldsymbol{q}^{\prime}\right)
$$

on both sides of the fermion loops $L^{\mp}$ in Equations (1719). After a lengthy but straightforward calculation (analogous to Lichtenstein et al. [26]), we obtain the following RGE in matrix form, where we denote by $\mathbb{P}, \mathbb{C}$ and $\mathbb{D}$ the matrices with respective entries $P_{R_{R^{\prime}}}, C_{R_{R^{\prime}}}$, and $D_{\boldsymbol{R} \boldsymbol{R}^{\prime}}$ (for the analogous equations in the SM-fRG framework, see [50, Equation (A3)]):

$$
\begin{aligned}
& \dot{\mathbb{P}}_{n_{1} n_{2} n_{3} n_{4}}(\boldsymbol{s})=\sum_{\ell_{1}, \ell_{2}} \hat{\mathbb{P}}\left[V_{n_{1} n_{2} \ell_{1} \ell_{2}}\right](\boldsymbol{s}) \mathbb{I}_{\ell_{1} \ell_{2}}^{-}(\boldsymbol{s}) \hat{\mathbb{P}}\left[V_{\ell_{2} \ell_{1} n_{3} n_{4}}\right](\boldsymbol{s}), \\
& \dot{\mathbb{C}}_{n_{1} n_{2} n_{3} n_{4}}(\boldsymbol{u})=2 \sum_{\ell_{1}, \ell_{2}} \hat{\mathbb{C}}\left[V_{n_{1} \ell_{2} n_{3} \ell_{1}}\right](\boldsymbol{u}) \mathbb{\mathbb { I }}_{\ell_{1} \ell_{2}}^{+}(\boldsymbol{u}) \hat{\mathbb{C}}\left[V_{\ell_{1} n_{2} \ell_{2} n_{4}}\right](\boldsymbol{u}), \\
& \dot{\mathbb{D}}_{n_{1} n_{2} n_{3} n_{4}}(\boldsymbol{t})=-2 \sum_{\ell_{1} \ell_{2}} \hat{\mathbb{D}}\left[V_{n_{1} \ell_{2} \ell_{1} n_{4}}\right](\boldsymbol{t}) \mathbb{I}_{\ell_{1} \ell_{2}}^{+}(\boldsymbol{t}) \hat{\mathbb{D}}\left[V_{\ell_{1} n_{2} n_{3} \ell_{2}}\right](\boldsymbol{t}) .
\end{aligned}
$$

Here, the loop matrices are given in terms of Equation (20) by

$$
\begin{aligned}
{\left[\mathbb{I}_{\ell_{1} \ell_{2}}^{-}(\boldsymbol{s})\right]_{R_{1} R_{2}} } & =\frac{1}{N} \sum_{\boldsymbol{q}} L_{\ell_{1} \ell_{2}}^{-}(\boldsymbol{s}-\boldsymbol{q}, \boldsymbol{q}) f_{\boldsymbol{R}_{1}}(\boldsymbol{q}) f_{\boldsymbol{R}_{2}}^{*}(\boldsymbol{q}), \\
{\left[\mathbb{L}_{\ell_{1} \ell_{2}}^{+}(\boldsymbol{u})\right]_{\boldsymbol{R}_{1} \boldsymbol{R}_{2}} } & =\frac{1}{N} \sum_{\boldsymbol{q}} L_{\ell_{1} \ell_{2}}^{+}(\boldsymbol{q}, \boldsymbol{q}-\boldsymbol{u}) f_{\boldsymbol{R}_{1}}(\boldsymbol{q}) f_{\boldsymbol{R}_{2}}^{*}(\boldsymbol{q}) .
\end{aligned}
$$

Finally, by substituting the decomposition (26) into the righthand sides of Equations (34-36) and performing the projections in the various channels (see section 3.3) we obtain a closed system of differential equations for the matrices $\mathbb{P}, \mathbb{C}$, and $\mathbb{D}$. Since for implementing these flow equations numerically, the form-factor expansion in Equation (33) has to be truncated appropriately (see the remarks in section 2, and for a detailed discussion, see [26]), the resulting technique is called "truncated-unity fRG" or “TUfRG”.

\subsection{Antisymmetry of Particle-Hole Terms}

We now come to a crucial observation which allows us to further reduce the number of relevant channels in the generalized TU-fRG equations [where the SU(2) symmetry has not been exploited]. In fact, the crossed and the direct particle-hole terms are directly related through Equation (19), and with the definitions $(24,25)$, this implies that

$$
V_{n_{1} n_{2} n_{3} n_{4}}^{(D)}\left(\boldsymbol{k}_{1}, \boldsymbol{k}_{2}, \boldsymbol{k}_{3}\right)=-V_{n_{1} n_{2} n_{4} n_{3}}^{(C)}\left(\boldsymbol{k}_{1}, \boldsymbol{k}_{2}, \boldsymbol{k}_{1}+\boldsymbol{k}_{2}-\boldsymbol{k}_{3}\right) .
$$

Further using the definitions $(28,29)$ and the property $(12)$, it follows that

$$
\begin{aligned}
\mathbb{D}_{n_{1} n_{2} n_{3} n_{4}}(\boldsymbol{t}) & =\hat{\mathbb{D}}\left[V_{n_{1} n_{2} n_{3} n_{4}}^{(D)}\right](\boldsymbol{t})=-\hat{\mathbb{C}}\left[V_{n_{1} n_{2} n_{4} n_{3}}^{(C)}\right](\boldsymbol{t}) \\
& =-\mathbb{C}_{n_{1} n_{2} n_{4} n_{3}}(\boldsymbol{t}) .
\end{aligned}
$$

Hence, the two matrices $\mathbb{C}$ and $\mathbb{D}$ are actually not independent of each other but simply related by the antisymmetry in the last two indices. As a consequence, one can show that the RGE (36) is in fact equivalent to Equation (35), and can therefore be discarded. We are thus left with only two RGE for the matrices $\mathbb{P}$ and $\mathbb{C}$ as given by Equations $(34,35)$. We remark that the antisymmetry property (40) is already well-established in SM-fRG works (see e.g., [50]).

\subsection{Projections}

It remains to perform the projections in Equations $(34,35)$ to obtain a closed system of RGE for the matrices $\mathbb{P}$ and $\mathbb{C}$. First, in the P-channel we have

$$
\begin{aligned}
\hat{\mathbb{P}}\left[V_{n_{1} n_{2} n_{3} n_{4}}\right]= & \hat{\mathbb{P}}\left[V_{n_{1} n_{2} n_{3} n_{4}}^{(0)}\right]+\mathbb{P}_{n_{1} n_{2} n_{3} n_{4}}+\hat{\mathbb{P}}\left[V_{n_{1} n_{2} n_{3} n_{4}}^{(C)}\right] \\
& +\hat{\mathbb{P}}\left[V_{n_{1} n_{2} n_{3} n_{4}}^{(D)}\right]
\end{aligned}
$$

which follows directly from the decomposition (26) and from the definition (27), i.e., $\mathbb{P}=\hat{\mathbb{P}}\left[V^{(P)}\right]$. The second-last term can be evaluated as follows:

$$
\begin{gathered}
\hat{P}\left[V_{n_{1} n_{2} n_{3} n_{4}}^{(C)}\right]_{\boldsymbol{R} \boldsymbol{R}^{\prime}}(\boldsymbol{s})= \\
\frac{1}{N^{2}} \sum_{\boldsymbol{k}, \boldsymbol{k}^{\prime}} f_{\boldsymbol{R}}(\boldsymbol{k}) f_{\boldsymbol{R}^{\prime}}^{*}\left(\boldsymbol{k}^{\prime}\right) V_{n_{1} n_{2} n_{3} n_{4}}^{(C)}\left(\boldsymbol{k}, \boldsymbol{s}-\boldsymbol{k}, \boldsymbol{s}-\boldsymbol{k}^{\prime}, \boldsymbol{k}^{\prime}\right) \\
=\frac{1}{N^{2}} \sum_{\boldsymbol{k}, \boldsymbol{k}^{\prime}} \sum_{\boldsymbol{R}_{1}, \boldsymbol{R}_{2}} f_{\boldsymbol{R}}(\boldsymbol{k}) f_{\boldsymbol{R}^{\prime}}^{*}\left(\boldsymbol{k}^{\prime}\right) f_{\boldsymbol{R}_{1}}^{*}(\boldsymbol{k}) f_{\boldsymbol{R}_{2}}\left(\boldsymbol{k}^{\prime}\right) C_{n_{1} n_{2} n_{3} n_{4}}^{\boldsymbol{R}_{1} \boldsymbol{R}_{2}}\left(\boldsymbol{k}+\boldsymbol{k}^{\prime}-\boldsymbol{s}\right) .
\end{gathered}
$$

We identify the form factors with plane-wave functions, $f_{\boldsymbol{R}}(\boldsymbol{k})=$ $\exp (-\mathrm{i} \boldsymbol{k} \cdot \boldsymbol{R})$, and perform a Fourier transformation of the matrix $\mathbb{C}$, i.e.,

$$
\begin{aligned}
& \mathbb{C}_{n_{1} n_{2} n_{3} n_{4}}(\boldsymbol{k})=\sum_{\boldsymbol{R}} \mathrm{e}^{-\mathrm{i} \boldsymbol{k} \cdot \boldsymbol{R}} \mathbb{C}_{n_{1} n_{2} n_{3} n_{4}}(\boldsymbol{R}), \\
& \mathbb{C}_{n_{1} n_{2} n_{3} n_{4}}(\boldsymbol{R})=\frac{1}{N} \sum_{\boldsymbol{k}} \mathrm{e}^{\mathrm{i} \boldsymbol{k} \cdot \boldsymbol{R}} \mathbb{C}_{n_{1} n_{2} n_{3} n_{4}}(\boldsymbol{k}) .
\end{aligned}
$$

Thus, we transform Equation (43) into

$$
\frac{1}{N^{2}} \sum_{\boldsymbol{k}, \boldsymbol{k}^{\prime}} \sum_{\boldsymbol{R}_{1}, \boldsymbol{R}_{2}, \boldsymbol{R}^{\prime \prime}} \mathrm{e}^{\mathrm{i} \boldsymbol{k} \cdot\left(-\boldsymbol{R}+\boldsymbol{R}_{1}-\boldsymbol{R}^{\prime \prime}\right)} \mathrm{e}^{\mathrm{i} \boldsymbol{k}^{\prime} \cdot\left(\boldsymbol{R}^{\prime}-\boldsymbol{R}_{2}-\boldsymbol{R}^{\prime \prime}\right)} \mathrm{e}^{\mathrm{i} \boldsymbol{\boldsymbol { R } ^ { \prime \prime }}} C_{n_{1} n_{2} n_{3} n_{4}}^{\boldsymbol{R}_{1} \boldsymbol{R}_{2}}\left(\boldsymbol{R}^{\prime \prime}\right) .
$$

Next, evaluating the sums over $\boldsymbol{k}$ and $\boldsymbol{k}^{\prime}$ yields two delta functions, which can be used to eliminate $\boldsymbol{R}_{1}$ and $\boldsymbol{R}_{2}$. Hence, we arrive at

$$
\hat{P}\left[V_{n_{1} n_{2} n_{3} n_{4}}^{(C)}\right]_{\boldsymbol{R} \boldsymbol{R}^{\prime}}(\boldsymbol{s})=\sum_{\boldsymbol{R}^{\prime \prime}} \mathrm{e}^{\mathrm{i} \boldsymbol{s} \cdot \boldsymbol{R}^{\prime \prime}} C_{n_{1} n_{2} n_{3} n_{4}}^{\boldsymbol{R}+\boldsymbol{R}^{\prime \prime}, \boldsymbol{R}^{\prime}-\boldsymbol{R}^{\prime \prime}}\left(\boldsymbol{R}^{\prime \prime}\right) .
$$

In particular, we note that this procedure (as used already in Wang et al. [46]) has reduced the double-wavevector sums of Equation (42) to a single sum over form-factor indices $\boldsymbol{R}^{\prime \prime}$. This sum is usually finite because the truncated form-factor matrices $C_{n_{1} n_{2} n_{3} n_{4}}^{\boldsymbol{R}_{1}, \boldsymbol{R}_{2}}(\boldsymbol{R})$ are nonzero only for a finite number of components $\boldsymbol{R}_{1}$ and $\boldsymbol{R}_{2}$. 
Similarly, we find [using the property (39) or (40)],

$$
\hat{P}\left[V_{n_{1} n_{2} n_{3} n_{4}}^{(D)}\right]_{\boldsymbol{R} \boldsymbol{R}^{\prime}}(\boldsymbol{s})=-\mathrm{e}^{\mathrm{i} \boldsymbol{s} \cdot \boldsymbol{R}^{\prime}} \sum_{\boldsymbol{R}^{\prime \prime}} \mathrm{e}^{\mathrm{i} \boldsymbol{s} \cdot \boldsymbol{R}^{\prime \prime}} C_{n_{1} n_{2} n_{4} n_{3}}^{\boldsymbol{R}+\boldsymbol{R}^{\prime \prime},-\boldsymbol{R}^{\prime \prime}}\left(\boldsymbol{R}^{\prime \prime}\right) .
$$

Next, we perform the projections in the $D$ channel. Again, we have

$$
\begin{aligned}
\hat{\mathbb{C}}\left[V_{n_{1} n_{2} n_{3} n_{4}}\right]= & \hat{\mathbb{C}}\left[V_{n_{1} n_{2} n_{3} n_{4}}^{(0)}\right]+\hat{\mathbb{C}}\left[V_{n_{1} n_{2} n_{3} n_{4}}^{(P)}\right]+\mathbb{C}_{n_{1} n_{2} n_{3} n_{4}} \\
& +\hat{\mathbb{C}}\left[V_{n_{1} n_{2} n_{3} n_{4}}^{(D)}\right]
\end{aligned}
$$

where we have used Equation (28). A straightforward calculation gives

$$
\hat{C}\left[V_{n_{1} n_{2} n_{3} n_{4}}^{(P)}\right]_{\boldsymbol{R} \boldsymbol{R}^{\prime}}(\boldsymbol{u})=\sum_{\boldsymbol{R}^{\prime \prime}} \mathrm{e}^{\mathrm{i} \boldsymbol{u} \cdot \boldsymbol{R}^{\prime \prime}} P_{n_{1} n_{2} n_{3} n_{4}}^{\boldsymbol{R}+\boldsymbol{R}^{\prime \prime}, \boldsymbol{R}^{\prime}-\boldsymbol{R}^{\prime \prime}}\left(\boldsymbol{R}^{\prime \prime}\right),
$$

as well as

$$
\hat{C}\left[V_{n_{1} n_{2} n_{3} n_{4}}^{(D)}\right]_{\boldsymbol{R} \boldsymbol{R}^{\prime}}(\boldsymbol{u})=-\sum_{\boldsymbol{R}^{\prime \prime}} \mathrm{e}^{\mathrm{i} \boldsymbol{u} \cdot \boldsymbol{R}^{\prime \prime}} C_{n_{1} n_{2} n_{4} n_{3}}^{\boldsymbol{R}-\boldsymbol{R}^{\prime}+\boldsymbol{R}^{\prime \prime}, \boldsymbol{R}^{\prime \prime}}\left(-\boldsymbol{R}^{\prime}\right) .
$$

In summary, in the non-SU(2)-symmetric case considered here, the channel-decomposed RGE for the matrices $\mathbb{P}$ and $\mathbb{C}$ are given by Equations (34), (35), (37), (38), (41), (47-51).

\section{ORBITAL VS. BAND BASIS}

\subsection{Transformation Laws}

For multiband systems, the vertex function can be represented either in the band basis or in the orbital basis, where these terms refer to the respective single-particle bases. Given the unitary matrix $U_{\sigma n}(\boldsymbol{k})$ which diagonalizes the single-particle Hamiltonian, these two representations of the vertex function are related as follows (see e.g., [6]):

$$
\begin{aligned}
& V_{n_{1} n_{2} n_{3} n_{4}}\left(\boldsymbol{k}_{1}, \boldsymbol{k}_{2}, \boldsymbol{k}_{3}\right)= \\
& \sum_{\sigma_{1}, \ldots, \sigma_{4}} U_{\sigma_{1} n_{1}}^{*}\left(\boldsymbol{k}_{1}\right) U_{\sigma_{2} n_{2}}^{*}\left(\boldsymbol{k}_{2}\right) V_{\sigma_{1} \sigma_{2} \sigma_{3} \sigma_{4}}\left(\boldsymbol{k}_{1}, \boldsymbol{k}_{2}, \boldsymbol{k}_{3}\right) \\
& U_{\sigma_{3} n_{3}}\left(\boldsymbol{k}_{3}\right) U_{\sigma_{4} n_{4}}\left(\boldsymbol{k}_{1}+\boldsymbol{k}_{2}-\boldsymbol{k}_{3}\right) .
\end{aligned}
$$

As mentioned before, we denote band indices by Latin letters and orbital indices by Greek letters. Later, we will consider the Rashba model as a two-band model, where the $\sigma_{i}$ can be identified with spin indices. In any case, given these two representations of the vertex function, we can also define the respective channel projections $\hat{P}\left[V_{\sigma_{1} \sigma_{2} \sigma_{3} \sigma_{4}}\right]$ or $\hat{P}\left[V_{n_{1} n_{2} n_{3} n_{4}}\right]$ as in section 2 (and similarly for $D$ and $C$ ). For deriving the transformation laws between these different matrices, we start from Equation (9) in the band basis, i.e.,

$\hat{P}\left[V_{n_{1} n_{2} n_{3} n_{4}}\right]_{\boldsymbol{R} \boldsymbol{R}^{\prime}}(\boldsymbol{s})=\frac{1}{N^{2}} \sum_{\boldsymbol{k}, \boldsymbol{k}^{\prime}} f_{\boldsymbol{R}}(\boldsymbol{k}) f_{\boldsymbol{R}^{\prime}}^{*}\left(\boldsymbol{k}^{\prime}\right) V_{n_{1} n_{2} n_{3} n_{4}}\left(\boldsymbol{k}, \boldsymbol{s}-\boldsymbol{k}, \boldsymbol{s}-\boldsymbol{k}^{\prime}\right)$.

We switch over to the spin basis using Equation (52), and then employ the converse relation (6) in the spin basis, i.e.,
$V_{\sigma_{1} \sigma_{2} \sigma_{3} \sigma_{4}}\left(\boldsymbol{k}, \boldsymbol{s}-\boldsymbol{k}, \boldsymbol{s}-\boldsymbol{k}^{\prime}\right)=\sum_{\boldsymbol{R}_{1}, \boldsymbol{R}_{2}} f_{\boldsymbol{R}_{1}}^{*}(\boldsymbol{k}) f_{\boldsymbol{R}_{2}}\left(\boldsymbol{k}^{\prime}\right) \hat{P}\left[V_{\sigma_{1} \sigma_{2} \sigma_{3} \sigma_{4}}\right]_{\boldsymbol{R}_{1} \boldsymbol{R}_{2}}(\boldsymbol{s})$

Thus, we arrive at the following transformation law for the particle-particle projection:

$$
\begin{gathered}
\hat{P}\left[V_{n_{1} n_{2} n_{3} n_{4}}\right]_{\boldsymbol{R} \boldsymbol{R}^{\prime}}(\boldsymbol{s})=\sum_{\sigma_{1}, \ldots, \sigma_{4}} \sum_{\boldsymbol{R}_{1}, \boldsymbol{R}_{2}}\left(\frac{1}{N} \sum_{\boldsymbol{k}} f_{\boldsymbol{R}}(\boldsymbol{k}) f_{\boldsymbol{R}_{1}}^{*}(\boldsymbol{k})\right. \\
\left.U_{\sigma_{2} n_{2}}^{*}(\boldsymbol{s}-\boldsymbol{k}) U_{\sigma_{1} n_{1}}^{*}(\boldsymbol{k})\right) \\
\times \hat{P}\left[V_{\sigma_{1} \sigma_{2} \sigma_{3} \sigma_{4}}\right]_{\boldsymbol{R}_{1} \boldsymbol{R}_{2}}(\boldsymbol{s})\left(\frac{1}{N} \sum_{\boldsymbol{k}^{\prime}} f_{\boldsymbol{R}_{2}}\left(\boldsymbol{k}^{\prime}\right) f_{\boldsymbol{R}^{\prime}}^{*}\left(\boldsymbol{k}^{\prime}\right)\right. \\
\left.U_{\sigma_{3} n_{3}}\left(\boldsymbol{s}-\boldsymbol{k}^{\prime}\right) U_{\sigma_{4} n_{4}}\left(\boldsymbol{k}^{\prime}\right)\right) .
\end{gathered}
$$

Defining the matrix

$$
U_{\sigma_{1} \sigma_{2} n_{1} n_{2}}^{R R^{\prime}}(\boldsymbol{s}):=\frac{1}{N} \sum_{\boldsymbol{k}} f_{\boldsymbol{R}}(\boldsymbol{k}) f_{\boldsymbol{R}^{\prime}}^{*}(\boldsymbol{k}) U_{\sigma_{1} n_{1}}(\boldsymbol{s}-\boldsymbol{k}) U_{\sigma_{2} n_{2}}(\boldsymbol{k}),
$$

we can write Equation (55) in matrix form as

$$
\begin{aligned}
\hat{\mathbb{P}}\left[V_{n_{1} n_{2} n_{3} n_{4}}\right](\boldsymbol{s})= & \sum_{\sigma_{1}, \ldots, \sigma_{4}}\left[\mathbb{U}_{\sigma_{2} \sigma_{1} n_{2} n_{1}}(\boldsymbol{s})\right]^{\dagger} \hat{\mathbb{P}}\left[V_{\sigma_{1} \sigma_{2} \sigma_{3} \sigma_{4}}\right](\boldsymbol{s}) \\
& \mathbb{U}_{\sigma_{3} \sigma_{4} n_{3} n_{4}}(\boldsymbol{s}) .
\end{aligned}
$$

The converse equation reads

$$
\begin{aligned}
\hat{\mathbb{P}}\left[V_{\sigma_{1} \sigma_{2} \sigma_{3} \sigma_{4}}\right](\boldsymbol{s})= & \sum_{n_{1}, \ldots, n_{4}} \mathbb{U}_{\sigma_{2} \sigma_{1} n_{2} n_{1}}(\boldsymbol{s}) \hat{\mathbb{P}}\left[V_{n_{1} n_{2} n_{3} n_{4}}\right](\boldsymbol{s}) \\
& {\left[\mathbb{U}_{\sigma_{3} \sigma_{4} n_{3} n_{4}}(\boldsymbol{s})\right]^{\dagger} . }
\end{aligned}
$$

Similarly, with the matrix

$$
X_{\sigma_{1} \sigma_{2} n_{1} n_{2}}^{\boldsymbol{R} \boldsymbol{R}^{\prime}}(\boldsymbol{u}):=\frac{1}{N} \sum_{\boldsymbol{k}} f_{\boldsymbol{R}}(\boldsymbol{k}) f_{\boldsymbol{R}^{\prime}}^{*}(\boldsymbol{k}) U_{\sigma_{1} n_{1}}(\boldsymbol{k}) U_{\sigma_{2} n_{2}}^{*}(\boldsymbol{k}-\boldsymbol{u}),
$$

we obtain the transformation laws

$$
\begin{aligned}
\hat{\mathbb{C}}\left[V_{n_{1} n_{2} n_{3} n_{4}}\right](\boldsymbol{u})= & \sum_{\sigma_{1}, \ldots, \sigma_{4}}\left[\mathbb{X}_{\sigma_{1} \sigma_{3} n_{1} n_{3}}(\boldsymbol{u})\right]^{\dagger} \hat{\mathbb{C}}\left[V_{\sigma_{1} \sigma_{2} \sigma_{3} \sigma_{4}}\right](\boldsymbol{u}) \\
& \mathbb{X}_{\sigma_{4} \sigma_{2} n_{4} n_{2}}(\boldsymbol{u}) \\
\hat{\mathbb{D}}\left[V_{n_{1} n_{2} n_{3} n_{4}}\right](\boldsymbol{t})= & \sum_{\sigma_{1}, \ldots, \sigma_{4}}\left[\mathbb{X}_{\sigma_{1} \sigma_{4} n_{1} n_{4}}(\boldsymbol{t})\right]^{\dagger} \hat{\mathbb{D}}\left[V_{\sigma_{1} \sigma_{2} \sigma_{3} \sigma_{4}}\right](\boldsymbol{t}) \\
& \mathbb{X}_{\sigma_{3} \sigma_{2} n_{3} n_{2}}(\boldsymbol{t})
\end{aligned}
$$

These transformation laws can be used to switch between the band basis and the orbital/spin basis in the TU-fRG scheme.

\subsection{RGE in Orbital Basis}

While in section 3.1 we have derived the channel-decomposed RGE in the band basis, one can analogously deduce the RGE 
in the orbital basis (see also [6, section III.E]). For example, the exchange propagator in the orbital basis,

$$
P_{\sigma_{1} \sigma_{2} \sigma_{3} \sigma_{4}}^{R \boldsymbol{R}^{\prime}}(\boldsymbol{s}):=\hat{P}\left[V_{\sigma_{1} \sigma_{2} \sigma_{3} \sigma_{4}}^{(P)}\right]_{\boldsymbol{R} \boldsymbol{R}^{\prime}}(\boldsymbol{s})
$$

fulfills the following RGE:

$$
\dot{\mathbb{P}}_{\sigma_{1} \sigma_{2} \sigma_{3} \sigma_{4}}(\boldsymbol{s})=\sum_{\tau_{1}, \ldots, \tau_{4}} \hat{\mathbb{P}}\left[V_{\sigma_{1} \sigma_{2} \tau_{1} \tau_{2}}\right](\boldsymbol{s}) \mathbb{L}_{\tau_{1} \tau_{2} \tau_{3} \tau_{4}}^{-}(\boldsymbol{s}) \hat{\mathbb{P}}\left[V_{\tau_{4} \tau_{3} \sigma_{3} \sigma_{4}}\right](\boldsymbol{s}),
$$

where the particle-particle loop in the orbital basis depends on four orbital indices and is given in terms of its counterpart in the band basis, Equation (37), by

$$
\mathbb{L}_{\tau_{1} \tau_{2} \tau_{3} \tau_{4}}^{-}(\boldsymbol{s})=\sum_{\ell_{1}, \ell_{2}} \mathbb{U}_{\tau_{1} \tau_{2} \ell_{1} \ell_{2}}(\boldsymbol{s}) \mathbb{L}_{\ell_{1} \ell_{2}}^{-}(\boldsymbol{s})\left[\mathbb{U}_{\tau_{3} \tau_{4} \ell_{1} \ell_{2}}(\boldsymbol{s})\right]^{\dagger} .
$$

Similarly, one can derive the RGE for the matrices $\mathbb{C}$ and $\mathbb{D}$ in the orbital basis.

\subsection{Discussion}

We conclude this section by a short comparison between the band basis and the orbital basis, focusing on practical aspects of the numerical implementation. The question which picture to choose arises in systems with more than one site per sublattice in the case of spin-rotational invariance, and even with one site per sublattice if spin-orbit coupling makes the single-particle Hamiltonian non-diagonal in the spins. While SM-fRG works like [46] use the orbital basis, previous TU-fRG studies like [28] and [32] were fomulated in the band basis.

On the one hand, the band basis diagonalizes the free Hamiltonian and the free Green function, and therefore the loop terms depend on only two band indices [see the explicit expressions (20-22)]. By contrast, in the orbital basis the Green function depends on two orbital indices, and correspondingly the loop terms depend on four orbital indices (see Equation 64). As a consequence, the CPU time required for a numerical implementation of the TU-fRG scales considerably with the number of orbitals $n_{\mathrm{o}}$ or the number of bands $n_{\mathrm{b}}$. Concretely, taking $n_{\mathrm{b}}=n_{\mathrm{o}}$, if we wish to compute the whole vertex function in the orbital basis, e.g., via Equation (63), then we need to (i) calculate all $n_{\mathrm{o}}^{4}$ entries of the exchange propagator matrices, (ii) perform $n_{\mathrm{o}}^{2}$ summations for calculating the loop terms via Equation (64), and (iii) perform the $n_{\mathrm{o}}^{4}$ summations on the righthand side of the RGE (63). Thus, the CPU time scales as $n_{\mathrm{o}}^{10}$, i.e., with the 10th power of the number of orbitals. By contrast, since in the band basis the loop terms are given explicitly by Equations (20-22), and since the right-hand sides of the RGE (34-36) require only a summation over two band indices, the CPU time scales only as $n_{\mathrm{b}}^{6}$, i.e., with the 6 th power of the number of bands.

On the other hand, the initial two-particle vertex is usually given in the orbital basis, where it often has a weak momentum dependence (corresponding to a local interaction in real space). By transforming the vertex into the band basis via Equation (52), it will typically acquire a complicated structure in momentum space (corresponding to a potentially complicated non-local form in real space). For the exchange propagators of the TU-fRG, this implies that a form-factor expansion in the orbital basis will lead to much faster convergence than in the band basis. In fact, we will confirm this last observation in the next section, where we will derive analytical expressions for the particle-particle exchange propagator in the Rashba model, both in the orbital basis and in the band basis [see Equations (69), (73), respectively]. It should also be noted that e.g., [32] discusses explicitly the convergence issues entailed by the projection of a longer-ranged initial interaction. Furthermore, at least the dominating parts of the bare interaction can usually be understood as a sum of terms with pairwise identical orbital indices. Hence, it may constitute a useful approximation to focus on the renormalization of these terms, which improves the scaling with $n_{\mathrm{o}}$.

\section{APPLICATION TO RASHBA MODEL}

We consider a general two-band model with the single-particle Hamiltonian given by

$$
H_{0}(\boldsymbol{k})=f(\boldsymbol{k}) \mathbb{1}+\boldsymbol{g}(\boldsymbol{k}) \cdot \boldsymbol{\sigma}
$$

where $f(\boldsymbol{k})$ and $g_{i}(\boldsymbol{k})(i=x, y, z)$ are real functions, and $\boldsymbol{\sigma}=$ $\left(\sigma_{x}, \sigma_{y}, \sigma_{z}\right)$ denotes the vector of Pauli matrices. We assume timereversal symmetry, which implies that $f(\boldsymbol{k})=f(-\boldsymbol{k})$ as well as $\boldsymbol{g}(\boldsymbol{k})=-\boldsymbol{g}(-\boldsymbol{k})$ (see e.g., [6, Appendix A2]). In particular, the Rashba model is recovered from the more general Equation (65) by setting

$$
f(\boldsymbol{k})=\hbar^{2}|\boldsymbol{k}|^{2} / 2 m^{*}, \quad g_{x}(\boldsymbol{k})=-k_{y}, \quad g_{y}(\boldsymbol{k})=k_{x}, \quad g_{z}(\boldsymbol{k})=0 .
$$

For this model with a local initial interaction, it turned out [6] that the particle-hole terms are negligible in the RG flow. The remaining particle-particle ladder could be resummed analytically in the spin basis [6, section III.E]. Correspondingly, we here neglect the matrix $\mathbb{C}$ and restrict attention to the remaining RGE (63) for the matrix

$$
\begin{aligned}
\mathbb{P}_{\sigma_{1} \sigma_{2} \sigma_{3} \sigma_{4}}(\boldsymbol{s})= & \hat{\mathbb{P}}\left[V_{\sigma_{1} \sigma_{2} \sigma_{3} \sigma_{4}}^{(P)}\right](\boldsymbol{s})=\hat{\mathbb{P}}\left[V_{\sigma_{1} \sigma_{2} \sigma_{3} \sigma_{4}}\right](\boldsymbol{s}) \\
& -\hat{\mathbb{P}}\left[V_{\sigma_{1} \sigma_{2} \sigma_{3} \sigma_{4}}^{(0)}\right](\boldsymbol{s}) .
\end{aligned}
$$

For an initial onsite interaction,

$$
V_{\sigma_{1} \sigma_{2} \sigma_{3} \sigma_{4}}^{(0)}\left(\boldsymbol{k}_{1}, \boldsymbol{k}_{2}, \boldsymbol{k}_{3}\right)=\frac{U}{2}\left(\delta_{\sigma_{1} \sigma_{3}} \delta_{\sigma_{2} \sigma_{4}}-\delta_{\sigma_{1} \sigma_{4}} \delta_{\sigma_{2} \sigma_{3}}\right)
$$

the analytical solution of this RGE reads as follows:

$$
P_{\sigma_{1} \sigma_{2} \sigma_{3} \sigma_{4}}^{R \boldsymbol{R}^{\prime}}(\boldsymbol{s})=-\frac{1}{2}\left(g_{\Lambda}(\boldsymbol{s})+U\right)\left(\delta_{\sigma_{1} \sigma_{3}} \delta_{\sigma_{2} \sigma_{4}}-\delta_{\sigma_{1} \sigma_{4}} \delta_{\sigma_{2} \sigma_{3}}\right) \delta_{\boldsymbol{R}, \mathbf{0}} \delta_{\boldsymbol{R}^{\prime}, \mathbf{0}}
$$

with the scale-dependent scalar function

$$
g_{\Lambda}(\boldsymbol{s})=-U\left(1+U \int_{\Lambda_{0}}^{\Lambda} B_{\Lambda^{\prime}}(\boldsymbol{s}) \mathrm{d} \Lambda^{\prime}\right)^{-1}
$$


Here, we have defined the auxiliary function

$$
\begin{aligned}
B(\boldsymbol{s}) & =\frac{1}{2} \sum_{\tau_{1}, \ldots, \tau_{4}}\left(\delta_{\tau_{1} \tau_{3}} \delta_{\tau_{2} \tau_{4}}-\delta_{\tau_{1} \tau_{4}} \delta_{\tau_{2} \tau_{3}}\right)\left[\mathbb{L}_{\tau_{1} \tau_{2} \tau_{3} \tau_{4}}^{-}(\boldsymbol{s})\right]_{\mathbf{0 0}} \\
& =\frac{1}{2} \sum_{\tau_{1}, \ldots, \tau_{4}}\left(\delta_{\tau_{1} \tau_{3}} \delta_{\tau_{2} \tau_{4}}-\delta_{\tau_{1} \tau_{4}} \delta_{\tau_{2} \tau_{3}}\right) \frac{1}{N} \sum_{\boldsymbol{k}} L_{\tau_{1} \tau_{2} \tau_{3} \tau_{4}}^{-}(\boldsymbol{s}-\boldsymbol{k}, \boldsymbol{k})
\end{aligned}
$$

which coincides with [6, Equation (178)]. Remarkably, the exchange propagator (69) in the spin basis remains local at any scale below the initial scale $\Lambda_{0}$.

Next, we transform Equation (69) into the band basis by means of Equation (57). Taking advantage of the formula (40) in Schober et al. [6], we arrive at

$$
\begin{aligned}
P_{n_{1} n_{2} n_{3} n_{4}}^{R \boldsymbol{R}^{\prime}}(\boldsymbol{s})= & -\frac{1}{8}(g(\boldsymbol{s})+U) \frac{1}{N^{2}} \sum_{\boldsymbol{k}, \boldsymbol{k}^{\prime}} f_{\boldsymbol{R}}(\boldsymbol{k}) f_{\boldsymbol{R}^{\prime}}^{*}\left(\boldsymbol{k}^{\prime}\right) \\
& {\left[n_{1} n_{3} \mathrm{e}^{\mathrm{i} \varphi\left(\boldsymbol{s}-\boldsymbol{k}^{\prime}\right)-\mathrm{i} \varphi(\boldsymbol{k})}+n_{2} n_{4} \mathrm{e}^{\mathrm{i} \varphi\left(\boldsymbol{k}^{\prime}\right)-\mathrm{i} \varphi(\boldsymbol{s}-\boldsymbol{k})}\right.} \\
& \left.-n_{1} n_{4} \mathrm{e}^{\mathrm{i} \varphi\left(\boldsymbol{k}^{\prime}\right)-\mathrm{i} \varphi(\boldsymbol{k})}-n_{2} n_{3} \mathrm{e}^{\mathrm{i} \varphi\left(\boldsymbol{s}-\boldsymbol{k}^{\prime}\right)-\mathrm{i} \varphi(\boldsymbol{s}-\boldsymbol{k})}\right],
\end{aligned}
$$

where $\varphi(\boldsymbol{k})$ denotes the polar angle of the vector $\boldsymbol{g}(\boldsymbol{k})$, and where the band indices $n_{i} \in\{-,+\}$ label the lower or upper band, respectively (see [6, section II]). Further using Equation (9), we read off the vertex function in the band basis as

$$
\begin{aligned}
V_{n_{1} \ldots n_{4}}\left(\boldsymbol{k}, \boldsymbol{s}-\boldsymbol{k}, \boldsymbol{s}-\boldsymbol{k}^{\prime}\right)= & -\frac{1}{8} g(\boldsymbol{s})\left[n_{1} n_{3} \mathrm{e}^{\mathrm{i} \varphi\left(\boldsymbol{s}-\boldsymbol{k}^{\prime}\right)-\mathrm{i} \varphi(\boldsymbol{k})}\right. \\
& +n_{2} n_{4} \mathrm{e}^{\mathrm{i} \varphi\left(\boldsymbol{k}^{\prime}\right)-\mathrm{i} \varphi(\boldsymbol{s}-\boldsymbol{k})} \\
& -n_{1} n_{4} \mathrm{e}^{\mathrm{i} \varphi\left(\boldsymbol{k}^{\prime}\right)-\mathrm{i} \varphi(\boldsymbol{k})} \\
& \left.-n_{2} n_{3} \mathrm{e}^{\mathrm{i} \varphi\left(\boldsymbol{s}-\boldsymbol{k}^{\prime}\right)-\mathrm{i} \varphi(\boldsymbol{s}-\boldsymbol{k})}\right]
\end{aligned}
$$

In particular, for $\boldsymbol{s}=\mathbf{0}$, this reduces to

$$
V_{n_{1} \ldots n_{4}}\left(\boldsymbol{k},-\boldsymbol{k},-\boldsymbol{k}^{\prime}\right)=\frac{1}{2} g(\mathbf{0}) \delta_{n_{1} n_{2}} \delta_{n_{3} n_{4}} n_{1} n_{4} \mathrm{e}^{\mathrm{i} \varphi\left(\boldsymbol{k}^{\prime}\right)-\mathrm{i} \varphi(\boldsymbol{k})},
$$

which is again consistent with the results of Schober et al. [6]. We note that the exchange propagator in the band basis, Equation (73), is not local anymore [in contrast to the expression (69) in the orbital basis]. Thus, in accordance with the remarks in section 4.3, a numerical implementation of the TU-fRG in the band basis would require one to keep track of a large number of form factors.

Finally, the above analytical solution for the Rashba model also gives some insights into the general advantages of the TUfRG technique. In fact, our expression (74) in the band basis shows a complicated momentum dependence, where for $\boldsymbol{s} \neq \mathbf{0}$ the vertex function does not only depend on the angular variables $\varphi(\boldsymbol{k})$ and $\varphi\left(\boldsymbol{k}^{\prime}\right)$. For reproducing the correct form of the effective interaction in a numerical implementation, it would therefore be necessary to take into account a sufficiently fine mesh of discrete wavevectors over the whole Brillouin zone. This, however, would be computationally demanding for an ordinary Fermi surface patching scheme (where the CPU time scales with the fourth power of the number of patches). By contrast, the TU-fRG scales only linearly with the number of Bloch momenta [26] and therefore allows for a much higher resolution of the momentum dependencies.

\section{CONCLUSION AND OUTLOOK}

We have reviewed the TU-fRG as a flexible and unbiased tool for investigating correlated electron systems, and we have adapted it for application to multiband systems with spinorbit coupling. In particular, we have defined the single-channel coupling functions and exchange propagators in the general case without $S U(2)$ symmetry. As a consequence of an antisymmetry relation, only two exchange propagators (which correspond to the particle-particle and the crossed particle-hole term) actually need to be considered. Furthermore, we have derived the corresponding flow equations, which are of a particularly simple form [see Equations (34-36), and compare them to the corresponding equations in the $\mathrm{SU}(2)$-symmetric case, i.e., [26, Equations (22-24)]]. In fact, these flow equations are analogous to the corresponding equations in the SM-fRG [50]. On the righthand side of the flow equations, projections between the different channels have to be performed, for which we have derived explicit expressions in section 3.3. We have also compared the different formulations of the TU-fRG in the band basis and the orbital basis. Finally, we have analytically solved the channeldecomposed RGE in the particle-particle channel for the Rashba model with a local interaction, whereby we have shown the consistency of this solution with the ladder resummation of Schober et al. [6].

To put this work into perspective, let us summarize the main advantages of the TU-fRG and outline possible future applications: First, in a numerical implementation the CPU time scales only linearly with the number of discrete Bloch momenta, which allows one to reach an extremely high momentum-space resolution. At the same time, the time-consuming part of calculating the right-hand sides of the flow equations can be parallelized efficiently on a large number of compute nodes [26]. For these reasons, the TU-fRG may be particularly advantageous in cases where Fermi surface patching with an insufficient momentum resolution influences the leading instability or the form of the effective interaction (see e.g., [55]). Furthermore, the speed-up gained from the efficient parallelization can be used to treat complicated multiband systems (for a proof of principle see [28]) or long-range interactions, which generally lead to sharp structures in momentum space [32]. Other possible future directions include the treatment of three-dimensional band structures (where usually, the implementation of Fermi surface patching is numerically too expensive), the investigation of frequency-dependent interaction vertices, or the consideration of self-energy feedback onto the flow of the two-particle vertex. As general advantages of the TU-fRG, we further mention its applicability in wide parameter ranges (in which it complements non-perturbative 
methods such as lattice QMC), and its unbiasedness with regard to different (and possibly competing) ordering tendencies [30].

With the present extension of the TU-fRG to non-SU(2)symmetric systems, we have further enlarged its range of applications to embrace the important class of spin-orbit coupled materials. These include non-centrosymmetric (and possibly topological) superconductors [5], Rashba semiconductors [18, 56], and Weyl semimetals [57]. In particular, the three-dimensional dispersion of Weyl semimetals has hindered so far a direct application of the fRG with Fermi surface patching, whereas their investigation using TU-fRG is feasible and currently underway. Thus, we expect the TU-fRG and its generalization presented here to play an important role in the quantitative description of correlated quantum materials.

\section{REFERENCES}

1. Mackenzie AP, Scaffidi T, Hicks CW, Maeno Y. Even odder after twenty-three years: the superconducting order parameter puzzle of $\mathrm{Sr}_{2} \mathrm{RuO}_{4}$. Quan Mater. (2017) 2:40. doi: 10.1038/s41535-017-0045-4

2. Sun HH, Jia JF. Detection of Majorana zero mode in the vortex. Quan Mater. (2017) 2:34. doi: 10.1038/s41535-017-0037-4

3. Xu N, Xu Y, Zhu J. Topological insulators for thermoelectrics. Quan Mater. (2017) 2:51. doi: 10.1038/s41535-017-0054-3

4. Winkler R. Spin-Orbit Coupling Effects in Two-Dimensional Electron and Hole Systems. Berlin; Heidelberg: Springer-Verlag (2003).

5. Smidman M, Salamon MB, Yuan HQ, Agterberg DF. Superconductivity and spin-orbit coupling in non-centrosymmetric materials: a review. Rep Prog Phys. (2017) 80:036501. doi: 10.1088/1361-6633/80/3/036501

6. Schober GAH, Giering KU, Scherer MM, Honerkamp C, Salmhofer M. Functional renormalization and mean-field approach to multiband systems with spin-orbit coupling: application to the Rashba model with attractive interaction. Phys Rev B (2016) 93:115111. doi: 10.1103/PhysRevB.93.115111

7. Lee JS, Schober GAH, Bahramy MS, Murakawa H, Onose Y, Arita R, et al. Optical response of relativistic electrons in the polar BiTeI semiconductor. Phys Rev Lett. (2011) 107:117401. doi: 10.1103/PhysRevLett.107.117401

8. Demkó L, Schober GAH, Kocsis V, Bahramy MS, Murakawa H, Lee JS, et al. Enhanced infrared magneto-optical response of the nonmagnetic semiconductor BiTeI driven by bulk Rashba splitting. Phys Rev Lett. (2012) 109:167401. doi: 10.1103/PhysRevLett.109.167401

9. Lutchyn RM, Sau JD, Das Sarma S. Majorana Fermions and a topological phase transition in semiconductor-superconductor heterostructures. Phys Rev Lett. (2010) 105:077001. doi: 10.1103/PhysRevLett.105.077001

10. Oreg Y, Refael G, von Oppen F. Helical liquids and Majorana bound states in quantum wires. Phys Rev Lett. (2010) 105:177002. doi: 10.1103/PhysRevLett.105.177002

11. Sinova J, Žutić I. New moves of the spintronics tango. Nat Mater. (2012) 11:368-71. doi: 10.1038/nmat3304

12. Wu CT, Anderson BM, Hsiao WH, Levin K. Majorana zero modes in spintronics devices. Phys Rev B (2017) 95:014519. doi: 10.1103/PhysRevB.95.014519

13. Rashba EI. Quantum nanostructures in strongly spin-orbit coupled two-dimensional systems. Phys Rev B (2012) 86:125319. doi: 10.1103/PhysRevB.86.125319

14. Schober GAH, Murakawa H, Bahramy MS, Arita R, Kaneko Y, Tokura Y, et al. Mechanisms of enhanced orbital dia- and paramagnetism: application to the Rashba semiconductor BiTeI. Phys Rev Lett. (2012) 108:247208. doi: 10.1103/ PhysRevLett.108.247208

15. Schwalbe S, Wirnata R, Starke R, Schober GAH, Kortus J. Ab initio electronic structure and optical conductivity of bismuth tellurohalides. Phys Rev B (2016) 94:205130. doi: 10.1103/PhysRevB.94.205130

\section{AUTHOR CONTRIBUTIONS}

All authors listed have made a substantial, direct, and intellectual contribution to the work, and approved it for publication.

\section{FUNDING}

This research was supported by the DFG grants HO 2422/10-1, 11-1, and 12-1 and by the DFG RTG 1995.

\section{ACKNOWLEDGMENTS}

We thank Christian J. Eckhardt, Julian Lichtenstein, Manfred Salmhofer, David Sánchez de la Peña, Michael M. Scherer, and Qianghua Wang for discussions.

16. Bahramy MS, Ogawa N. Bulk Rashba semiconductors and related quantum phenomena. Adv Mater. (2017) 29:1605911. doi: 10.1002/adma.2016 05911

17. Picozzi S. Ferroelectric Rashba semiconductors as a novel class of multifunctional materials. Front Phys. (2014) 2:10. doi: 10.3389/fphy.2014.00010

18. Liebmann M, Rinaldi C, Di Sante D, Kellner J, Pauly C, Wang RN, et al. Giant Rashba-type spin splitting in ferroelectric GeTe(111). Adv Mater. (2016) 28:560-5. doi: 10.1002/adma.201503459

19. Datta S, Das B. Electronic analog of the electro-optic modulator. Appl Phys Lett. (1990) 56:665-7. doi: 10.1063/1.102730

20. Koo HC, Kwon JH, Eom J, Chang J, Han SH, Johnson M. Control of spin precession in a spin-injected field effect transistor. Science (2009) 325:1515-8. doi: 10.1126/science. 1173667

21. Ciftja O. Electric field controlled spin interference in a system with Rashba spin-orbit coupling. AIP Adv. (2016) 6:055217. doi: 10.1063/1.4952756

22. Dzero M, Sun K, Coleman P, Galitski V. Theory of topological Kondo insulators. Phys Rev B. (2012) 85:045130. doi: 10.1103/PhysRevB.85. 045130

23. Isobe $\mathrm{H}, \mathrm{Fu} \mathrm{L}$. Theory of interacting topological crystalline insulators. Phys Rev B (2015) 92:081304. doi: 10.1103/PhysRevB.92.081304

24. Gor'kov LP, Rashba EI. Superconducting 2D system with lifted spin degeneracy: mixed singlet-triplet state. Phys Rev Lett. (2001) 87:037004. doi: 10.1103/PhysRevLett.87.037004

25. Narayan V, Nguyen TA, Mansell R, Ritchie D, Mussler G. Interplay of spinorbit coupling and superconducting correlations in germanium telluride thin films. Rapid Res Lett. (2016) 10:253-9. doi: 10.1002/pssr.201510430

26. Lichtenstein J, de la Peña DS, Rohe D, Di Napoli E, Honerkamp C Maier SA. High-performance functional renormalization group calculations for interacting fermions. Comput Phys Commun. (2017) 213:100-10. doi: 10.1016/j.cpc.2016.12.013

27. Lichtenstein J, Winkelmann J, Sánchez de la Peña D, Vidović T, Di Napoli E. Parallel adaptive integration in high-performance functional renormalization group computations. In: Di Napoli E, Hermanns MA, Iliev H, Lintermann A, Peyser A, editors. High-Performance Scientific Computing. JHPCS 2016. Lecture Notes in Computer Science, Vol. 10164. Cham: Springer International Publishing (2017). p. 170-84.

28. Sánchez de la Peña D, Lichtenstein J, Honerkamp C. Competing electronic instabilities of extended Hubbard models on the honeycomb lattice: a functional renormalization group calculation with high-wave-vector resolution. Phys Rev B (2017) 95:085143. doi: 10.1103/PhysRevB.95.085143

29. Kopietz P, Bartosch L, Schütz F. Introduction to the Functional Renormalization Group. Berlin; Heidelberg: Springer (2010).

30. Metzner W, Salmhofer M, Honerkamp C, Meden V, Schönhammer K. Functional renormalization group approach to correlated fermion systems. Rev Mod Phys. (2012) 84:299. doi: 10.1103/RevModPhys.84.299 
31. Platt C, Hanke W, Thomale R. Functional renormalization group for multi-orbital Fermi surface instabilities. Adv Phys. (2013) 62:453-562. doi: 10.1080/00018732.2013.862020

32. Sánchez de la Peña D, Lichtenstein J, Honerkamp C, Scherer MM. Antiferromagnetism and competing charge instabilities of electrons in strained graphene from Coulomb interactions. Phys Rev B (2017) 96:205155. doi: 10.1103/PhysRevB.96.205155

33. Giering KU, Salmhofer M. Self-energy flows in the twodimensional repulsive Hubbard model. Phys Rev B (2012) 86:245122. doi: 10.1103/PhysRevB.86.245122

34. Eberlein A, Metzner W. Superconductivity in the twodimensional $t$ - $t^{\prime}$-Hubbard model. Phys Rev B (2014) 89:035126. doi: 10.1103/PhysRevB.89.035126

35. Lichtenstein J, Maier SA, Honerkamp C, Platt C, Thomale R, Andersen OK, et al. Functional renormalization group study of an eight-band model for the iron arsenides. Phys Rev B (2014) 89:214514. doi: 10.1103/PhysRevB.89. 214514

36. Classen L, Herbut IF, Janssen L, Scherer MM. Mott multicriticality of Dirac electrons in graphene. Phys Rev B (2015) 92:035429. doi: 10.1103/PhysRevB.92.035429

37. Lang TC, Meng ZY, Scherer MM, Uebelacker S, Assaad FF, Muramatsu A, et al. Antiferromagnetism in the Hubbard Model on the BernalStacked Honeycomb Bilayer. Phys Rev Lett. (2012) 109:126402. doi: 10.1103/PhysRevLett.109.126402

38. de la Peña DS, Scherer MM, Honerkamp C. Electronic instabilities of the AA-Honeycomb Bilayer. Ann Phys. (2014) 526:366-71. doi: $10.1002 /$ andp. 201400088

39. Scherer MM, Uebelacker S, Scherer DD, Honerkamp C. Interacting electrons on trilayer honeycomb lattices. Phys Rev B (2012) 86:155415. doi: 10.1103/PhysRevB.86.155415

40. Maier SA, Eberlein A, Honerkamp C. Functional renormalization group for commensurate antiferromagnets: Beyond the mean-field picture. Phys Rev B (2014) 90:035140. doi: 10.1103/PhysRevB.90.035140

41. Scherer DD, Scherer MM, Khaliullin G, Honerkamp C, Rosenow B. Unconventional pairing and electronic dimerization instabilities in the doped Kitaev-Heisenberg model. Phys Rev B (2014) 90:045135. doi: 10.1103/PhysRevB.90.045135

42. Husemann C, Salmhofer M. Efficient parametrization of the vertex function, $\Omega$ scheme, and the $t, t^{\prime}$ Hubbard model at van Hove filling. Phys Rev B (2009) 79:195125. doi: 10.1103/PhysRevB.79.195125

43. Husemann C, Giering KU, Salmhofer M. Frequency-dependent vertex functions of the $\left(t, t^{\prime}\right)$ Hubbard model at weak coupling. Phys Rev B (2012) 85:075121. doi: 10.1103/PhysRevB.85.075121

44. Eberlein A, Metzner W. Parametrization of Nambu vertex in a singlet superconductor. Prog Theor Phys. (2010) 124:471. doi: 10.1143/PTP.124.471

45. Maier SA, Ortloff J, Honerkamp C. Multiorbital effects in the functional renormalization group: A weak-coupling study of the Emery model. Phys Rev $B$ (2013) 88:235112. doi: 10.1103/PhysRevB.88.235112
46. Wang WS, Xiang YY, Wang QH, Wang F, Yang F, Lee DH. Functional renormalization group and variational Monte Carlo studies of the electronic instabilities in graphene near $\frac{1}{4}$ doping. Phys Rev B (2012) 85:035414. doi: 10.1103/PhysRevB.85.035414

47. Salmhofer M, Honerkamp C. Fermionic renormalization group flows: technique and theory. Prog Theor Phys. (2001) 105:1-35. doi: 10.1143/PTP.105.1

48. Eberlein A. Self-energy effects in functional renormalization group flows of the two-dimensional $t$ - $t^{\prime}$ Hubbard model away from van Hove filling. Phys Rev B (2015) 92:235146. doi: 10.1103/PhysRevB.92.235146

49. Vilardi D, Taranto C, Metzner W. Non-separable frequency dependence of two-particle vertex in interacting fermion systems. Phys Rev B (2017) 96:235110. doi: 10.1103/PhysRevB.96.235110

50. Xiang YY, Wang WS, Wang QH, Lee DH. Topological superconducting phase in the vicinity of ferromagnetic phases. Phys Rev B (2012) 86:024523. doi: 10.1103/PhysRevB.86.024523

51. Wang WS, Yang Y, Wang QH. Triplet $f$-wave pairing in SrPtAs. Phys Rev B (2014) 90:094514. doi: 10.1103/PhysRevB.90.094514

52. Yang Y, Wang WS, Xiang YY, Li ZZ, Wang QH. Triplet pairing and possible weak topological superconductivity in $\mathrm{BiS}_{2}$-based superconductors. Phys Rev B (2013) 88:094519. doi: 10.1103/PhysRevB.88.094519

53. Yang Y, Wang WS, Liu JG, Chen H, Dai JH, Wang QH. Superconductivity in doped $\mathrm{Sr}_{2} \mathrm{IrO}_{4}$ : a functional renormalization group study. Phys Rev B (2014) 89:094518. doi: 10.1103/PhysRevB.89.094518

54. Eckhardt CJ, Schober GAH, Ehrlich J, Honerkamp C. Truncatedunity parquet equations: Application to the repulsive Hubbard model. arXiv: $1802.09797 \mathrm{v} 2$

55. Volpez Y, Scherer DD, Scherer MM. Electronic instabilities of the extended Hubbard model on the honeycomb lattice from functional renormalization. Phys Rev B (2016) 94:165107. doi: 10.1103/PhysRevB.94.165107

56. Ishizaka K, Bahramy MS, Murakawa H, Sakano M, Shimojima T, Sonobe T, et al. Giant Rashba-type spin splitting in bulk BiTeI. Nat Mater. (2011) 10:521-6. doi: 10.1038/nmat3051

57. Zyuzin AA, Burkov AA. Topological response in Weyl semimetals and the chiral anomaly. Phys Rev B (2012) 86:115133. doi: 10.1103/PhysRevB.86.115133

Conflict of Interest Statement: The authors declare that the research was conducted in the absence of any commercial or financial relationships that could be construed as a potential conflict of interest.

Copyright (C) 2018 Schober, Ehrlich, Reckling and Honerkamp. This is an open-access article distributed under the terms of the Creative Commons Attribution License (CC $B Y)$. The use, distribution or reproduction in other forums is permitted, provided the original author(s) and the copyright owner are credited and that the original publication in this journal is cited, in accordance with accepted academic practice. No use, distribution or reproduction is permitted which does not comply with these terms. 